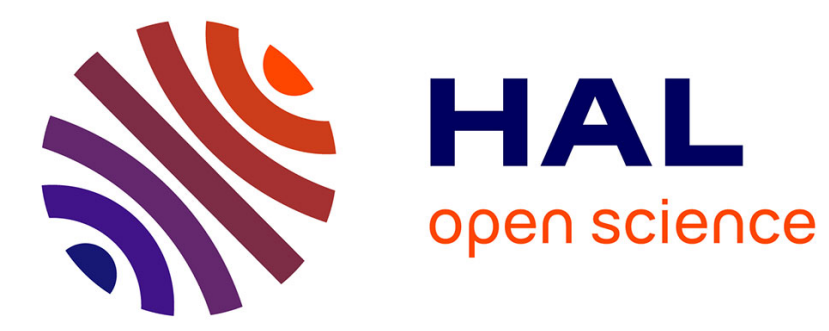

\title{
TWO MORE CHARACTERIZATIONS OF K-TRIVIALITY
}

Noam Greenberg, Joseph S Miller, Benoit Monin, Daniel Turetsky

\section{To cite this version:}

Noam Greenberg, Joseph S Miller, Benoit Monin, Daniel Turetsky. TWO MORE CHARACTERI-

ZATIONS OF K-TRIVIALITY. Notre Dame Journal of Formal Logic, 2016. hal-01397290

\section{HAL Id: hal-01397290 \\ https://hal.science/hal-01397290}

Submitted on 15 Nov 2016

HAL is a multi-disciplinary open access archive for the deposit and dissemination of scientific research documents, whether they are published or not. The documents may come from teaching and research institutions in France or abroad, or from public or private research centers.
L'archive ouverte pluridisciplinaire HAL, est destinée au dépôt et à la diffusion de documents scientifiques de niveau recherche, publiés ou non, émanant des établissements d'enseignement et de recherche français ou étrangers, des laboratoires publics ou privés. 


\title{
TWO MORE CHARACTERIZATIONS OF $K$-TRIVIALITY
}

\author{
NOAM GREENBERG, JOSEPH S. MILLER, BENOIT MONIN, AND DANIEL TURETSKY
}

\begin{abstract}
We give two new characterizations of $K$-triviality. We show that if for all $Y$ such that $\Omega$ is $Y$-random, $\Omega$ is $(Y \oplus A)$-random, then $A$ is $K$-trivial. The other direction was proved by Stephan and $Y u$, giving us the first titular characterization of $K$-triviality and answering a question of Yu. We also prove that if $A$ is $K$-trivial, then for all $Y$ such that $\Omega$ is $Y$-random, $(Y \oplus A) \equiv_{\mathrm{LR}} Y$. This answers a question of Merkle. The other direction is immediate, so we have the second characterization of $K$-triviality.

The proof of the first characterization uses a new cupping result. We prove that if $A \not \mathrm{LR}_{\mathrm{LR}} B$, then for every set $X$ there is a $B$-random set $Y$ such that $X$ is computable from $Y \oplus A$.
\end{abstract}

\section{Preliminaries}

We assume that the reader is familiar with basic notions from computability theory and effective randomness. For more information on these topics, we recommend either Nies [10] or Downey and Hirschfeldt [3].

The $K$-trivial sets have played an important role in the development of effective randomness. A set $A \in 2^{\omega}$ is $K$-trivial if $K\left(A\lceil n) \leq^{+} K(n)\right.$, where $K$ denotes prefix-free Kolmogorov complexity. Chaitin [1] proved that such sets are always $\Delta_{2}^{0}$, while Solovay [15] constructed a noncomputable $K$-trivial set. While these results date back to the 1970s, the importance of $K$-triviality did not become apparent until the 2000s, when several nontrivial characterizations were discovered. In particular:

Theorem 1.1 (Nies [9]; Hirschfeldt, Nies, and Stephan [5]). The following are equivalent for a set $A \in 2^{\omega}$ :

(a) $A$ is $K$-trivial,

(b) $A$ is low for $K: K^{A}(n) \geq^{+} K(n)$,

(c) $A$ is low for randomness: every random set is $A$-random, ${ }^{1}$

(d) $A$ is a base for randomness: there is an $A$-random set $X \geq_{\mathrm{T}} A$.

Nies [9] generalized (c) to LR-reducibility: we write $A \leq_{\mathrm{LR}} B$ to mean that every $B$-random set is $A$-random. In particular, $A \leq_{\mathrm{LR}} \emptyset$ means that $A$ is low for randomness (hence $K$-trivial).

Much more has been proved about the $K$-trivial sets, including many other characterizations. But we will only need one other fact. If $X$ is random, then we

Date: March 25, 2015.

2010 Mathematics Subject Classification. Primary 03D32; Secondary 68Q30.

This work was done while the authors were visiting the Research Centre Whiritoa in New Zealand, at the generous invitation of André Nies. Greenberg was supported by the Marsden Fund, a Rutherford Discovery Fellowship, and a Turing Research Fellowship "Mind, Mechanism and Mathematics" from the John Templeton Foundation. Turetsky was supported by the Austrian Science Fund FWF project I 1238.

${ }^{1}$ Throughout this paper, we consistently use random to mean Martin-Löf random. 
say that $Y$ is low for $X$ if $X$ is $Y$-random. This notion was introduced in [5], where it is shown that a set is $K$-trivial if and only if it is $\Delta_{2}^{0}$ and low for Chaitin's $\Omega$. However, many other sets are low for $\Omega$; for example, every 2-random set. A more recent result regarding $K$-triviality and lowness for $\Omega$ was used by Stephan and $\mathrm{Yu}$ to prove one direction of our first characterization (see the discussion before Proposition 3.2).

Theorem 1.2 (Simpson and Stephan [14, Theorem 3.11]). If $S$ has PA degree and is low for $\Omega$, then $S$ computes every $K$-trivial.

In addition to these facts about the $K$-trivial sets, we will use several fairly well-known theorems from effective randomness. Van Lambalgen's theorem [16] says that $X \oplus Y$ is random if and only if $X$ is random and $Y$ is $X$-random. Two applications allow us to show that if $X$ is random and $Y$ is $X$-random, then $X$ is $Y$-random. Every set is computable from some random set. Relativizing this to $X$ :

Theorem 1.3 (Kučera [7]; Gács [4]). For any sets $X$ and $C$, there is an $X$-random set $Y$ such that $C \leq_{\mathrm{T}} Y \oplus X$.

Any random set Turing below a $Z$-random set is also $Z$-random. Relativizing this to $Y$ :

Theorem 1.4 (Miller and $\mathrm{Yu}\left[8\right.$, Theorem 4.3]). Assume that $X \leq_{\mathrm{T}} W \oplus Y, X$ is $Y$-random, and $W$ is $Z \oplus Y$-random. Then $X$ is $Z \oplus Y$-random.

Finally, we will use the relativized form of the "randomness preservation" basis theorem:

Theorem 1.5 (Downey, Hirschfeldt, Miller, Nies [2]; Reimann and Slaman [13]). If $W$ is $Y$-random and $P$ is a nonempty $\Pi_{1}^{0}[Y]$ class, then there is a set $S \in P$ that is low for $W$.

\section{Cupping with $B$-RAndom sets}

As promised in the abstract, we prove the following cupping result.

Theorem 2.1. Assume that $A \not_{\mathrm{LR}} B$. Then for any set $X$, there is a $B$-random set $Y$ such that $X \leq_{\mathrm{T}} Y \oplus A$ (in fact, we make $Y$ weakly 2-random relative to $B$ ).

Our proof uses a result of Kjos-Hanssen. We state it here in a slightly stronger form than he stated it, though without adding any essential content.

Theorem 2.2 (Kjos-Hanssen [6]). $A \not \Varangle_{\mathrm{LR}} B$ if and only if there is a $\Sigma_{1}^{0}[A]$ class $U$ of measure less than one that intersects every positive measure $\Pi_{1}^{0}[B]$ class. Furthermore, for any $\varepsilon>0$, we can ensure that $\lambda(U)<\varepsilon$.

Kjos-Hanssen showed that $A \leq_{\mathrm{LR}} B$ if and only if each $\Pi_{1}^{0}[A]$ class of positive measure has a $\Pi_{1}^{0}[B]$ subclass of positive measure. ${ }^{2}$ Taking the contrapositive: $A \nless_{\mathrm{LR}} B$ if and only if there is a $\Pi_{1}^{0}[A]$ class $T$ of positive measure that does not have a positive measure $\Pi_{1}^{0}[B]$ subclass. So $U=2^{\omega} \backslash T$ would be a $\Sigma_{1}^{0}[A]$ class of measure less than one that intersects every positive measure $\Pi_{1}^{0}[B]$ class.

The fact that $U$ can be taken to have arbitrarily small measure also follows from the work in [6]. We use this fact below, so for completness, we sketch the argument. Assume that $A \not \mathbf{L}_{\mathrm{LR}} B$. So there is a $B$-random set $X$ that is not $A$-random. Let

\footnotetext{
${ }^{2}$ This partial relativization of [6, Theorem 2.10] is stated in the proof of [6, Theorem 3.2].
} 
$U$ be a $\Sigma_{1}^{0}[A]$ class containing every non- $A$-random set. We may assume, of course, that the measure of $U$ is as small as we like. Let $P$ be a positive measure $\Pi_{1}^{0}[B]$ class. Relativizing a result of Kučera [7], every $B$-random set has a tail in $P$, so there is a tail $Y$ of $X$ in $P$. But $Y$ is not $A$-random, so $Y \in U{ }^{3}$

We need some basic notation for the proof of Theorem 2.1. If $P \subseteq 2^{\omega}$ is measurable and $\sigma \in 2^{<\omega}$, let $\lambda(P \mid \sigma)$ denote the relative measure of $P$ in $[\sigma]$, i.e., $\lambda(P \cap[\sigma]) / \lambda([\sigma])$. If $\sigma \in 2^{<\omega}$ and $W \subseteq 2^{<\omega}$, let $\sigma W=\{\sigma \tau: \tau \in W\}$.

Proof of Theorem 2.1. Suppose that $A \Varangle_{\mathrm{LR}} B$. By Theorem 2.2, there is a $\Sigma_{1}^{0}[A]$ class $U$ such that $\lambda(U)<0.1$ and $U$ intersects every positive measure $\Pi_{1}^{0}[B]$ class. Let $W$ be an $A$-c.e. prefix-free set of strings such that $U=[W]^{\prec}$.

Let $X$ be any set. We will construct $Y=X(0) \sigma_{0} X(1) \sigma_{1} X(2) \sigma_{2} \cdots$ such that each $\sigma_{i} \in W$. In this way, it is clear that $X \leq_{\mathrm{T}} Y \oplus A$. To ensure that $Y$ is weakly 2-random relative to $B$, we build it inside a nested sequence of $\Pi_{1}^{0}[B]$ classes of positive measure. The following claim will let us hit $W$ and code the next bit of $X$ while staying inside the current $\Pi_{1}^{0}[B]$ class.

Claim. For any string $\sigma \in 2^{<\omega}$ and any $\Pi_{1}^{0}[B]$ class $P$ such that $\lambda(P \mid \sigma)>0.1$, there is a $\tau \succeq \sigma$ such that $\tau \in \sigma W$ and $\lambda(P \mid \tau) \geq 0.8$.

Proof. We first extend $\sigma$ to a string $\rho$ that has no prefix in $\sigma W$ and such that $\lambda(P \mid \rho)>0.9$. Let $Q=2^{\omega} \backslash[\sigma W]^{\prec}$. As $\lambda(Q \mid \sigma)>0.9$ and $\lambda(P \mid \sigma)>0.1$, we have $\lambda(Q \cap P \mid \sigma)>0$. By the Lebesgue density theorem, there is a $\rho \succeq \sigma$ such that $\lambda(Q \cap P \mid \rho)>0.9$. In particular, $\lambda(P \mid \rho)>0.9$ and $\lambda(Q \mid \rho)>0.9$; the latter implies that $\rho$ cannot have a prefix in $\sigma W$.

We now extend $\rho$ to a string $\tau$ satisfying the claim: $\tau \in \sigma W$ and $\lambda(P \mid \tau) \geq 0.8$. Consider the $\Pi_{1}^{0}(B)$ class $\widetilde{P}=\{X \in P \cap[\rho]:(\forall n \geq|\rho|) \lambda(P|X| n) \geq 0.8\}$. In words, $\widetilde{P}$ is the subclass of $P \cap[\rho]$ in which we remove every basic neighborhood inside $[\rho]$ where the relative measure of $P$ drops below 0.8 . It is not hard to show that we remove at most 0.8 from the relative measure of $P \cap[\rho]$ inside $[\rho]$ (consider the antichain of maximal basic neighborhoods that are removed). But $\lambda(P \mid \rho)>0.9$, so $\lambda(\widetilde{P} \mid \rho)>0.1$. In particular, $\widetilde{P}$ is a positive measure subclass of $[\sigma]$, so by the choice of $U=[W]^{\prec}$, it must be the case that $[\sigma W]^{\prec}$ intersects $\widetilde{P}$. Take $\tau \in \sigma W$ such that $\widetilde{P} \cap[\tau] \neq \emptyset$. By the definition of $\widetilde{P}$, we have $\lambda(P \mid \tau) \geq 0.8$. $\diamond$

We are ready to construct $Y$. We will construct it as the limit of a sequence $\tau_{0} \preceq \tau_{1} \preceq \tau_{2} \preceq \cdots$ of strings, while staying inside a decreasing sequence $P_{0} \supseteq$ $P_{1} \supseteq P_{2} \supseteq \cdots$ of $\Pi_{1}^{0}[B]$ classes. Let $P_{0}=2^{\omega}$ and let $\tau_{0}$ be the empty string. We start stage $n$ of the construction with a $\Pi_{1}^{0}[B]$ class $P_{n}$ and a string $\tau_{n}=$ $X(0) \sigma_{0} X(1) \cdots X(n-1) \sigma_{n-1}$ such that

$$
\lambda\left(P_{n} \mid \tau_{n} X(n)\right)>0.1 \text {. }
$$

(Note that this is true at stage 0.) First, we want to make progress towards $Y$ being weakly 2-random relative to $B$. Let $\bigcup_{m \in \omega} R_{m}$ be the $n$th $\Sigma_{2}^{0}[B]$ class of measure one, where $R_{0} \subseteq R_{1} \subseteq R_{2} \subseteq \cdots$ is a nested sequence of $\Pi_{1}^{0}[B]$ classes. Pick $m$ large enough that $\lambda\left(P_{n} \cap R_{m} \mid \tau_{n} X(n)\right)>0.1$ and let $P_{n+1}=P_{n} \cap R_{m}$. So as long as we ensure that $Y \in P_{n+1}$, we have ensured that $Y$ is in the $n$th $\Sigma_{2}^{0}[B]$ class of measure

\footnotetext{
${ }^{3}$ In fact, $U \cap P$ has positive measure. Choose $\sigma \in 2^{<\omega}$ such that $Y \in[\sigma] \subseteq U$. Then $\widetilde{P}=P \cap[\sigma] \subseteq P \cap U$ is a $\Pi_{1}^{0}[B]$ class. Since it contains $Y$, which is $B$-random, it cannot have measure zero.
} 
one. Now apply the claim to get $\tau_{n+1} \succeq \tau_{n} X(n)$ such that $\lambda\left(P_{n+1} \mid \tau_{n+1}\right) \geq 0.8$ and $\tau_{n+1} \in \tau_{n} X(n) W$. Let $\sigma_{n}$ be the string for which $\tau_{n+1}=\tau_{n} X(n) \sigma_{n}$; in particular, $\sigma_{n} \in W$. Note that $\lambda\left(P_{n+1} \mid \tau_{n+1} X(n+1)\right) \geq 0.6>0.1$, so $(\star)$ holds at stage $n+1$.

Let $Y=\bigcup_{n \in \omega} \tau_{n}=X(0) \sigma_{0} X(1) \sigma_{1} X(2) \sigma_{2} \cdots$. As promised, each $\sigma_{i}$ is in $W$, so $X \leq_{\mathrm{T}} Y \oplus A$. By construction, $P_{0} \supseteq P_{1} \supseteq P_{2} \supseteq \cdots$, and each $\tau_{n}$ can be extended to an element of $P_{n}$. Therefore, $Y \in \bigcap_{n \in \omega} P_{n}$. This ensures that $Y$ is in every $\Sigma_{2}^{0}[B]$ class of measure one, so $Y$ is weakly 2 -random relative to $B$.

\section{LOW FOR $X$ PRESERVING}

Definition 3.1. Let $X$ be random. A set $A$ is low for $X$ preserving if for all $Y$,

$$
Y \text { is low for } X \Longrightarrow Y \oplus A \text { is low for } X \text {. }
$$

This notion was recently introduced by Yu Liang, who called it absolutely low for $X$. Stephan and Yu proved that every $K$-trivial is low for $\Omega$ preserving (see $[11$, Fact 1.8]). Yu asked if the converse is true: if a set is low for $\Omega$ preserving, is it $K$-trivial? We show that, in fact, this holds in general.

Proposition 3.2. If $X$ is random, then low for $X$ preserving implies $K$-triviality.

Proof. Assume that $A$ is low for $X$ preserving.

First, we claim that $A \leq_{\mathrm{LR}} X$. If not, then Theorem 2.1 gives us an $X$-random set $Y$ such that $X \leq_{\mathrm{T}} Y \oplus A$. By Van Lambalgen's theorem, $X$ is $Y$-random. But $X \leq_{\mathrm{T}} Y \oplus A$ implies that $X$ is not $(Y \oplus A)$-random. This contradicts the assumption that $A$ is low for $X$ preserving. Therefore, $A \leq_{\mathrm{LR}} X$.

By Theorem 1.3, there is an $X$-random set $Y$ such that $A \leq_{\mathrm{T}} Y \oplus X$. Because $A$ is low for $X$ preserving, we have that $X$ is $(Y \oplus A)$-random. Furthermore, because $Y$ is $X$-random and $A \leq_{\mathrm{LR}} X$, we know that $Y$ is $A$-random. Therefore, by Van Lambalgen's theorem, $Y \oplus X$ is $A$-random. But $Y \oplus X \operatorname{computes} A$, so $A$ is a base for randomness. Therefore, it is $K$-trivial (see Theorem 1.1).

Together with the result of Stephan and $\mathrm{Yu}$, we get a new characterization of $K$-triviality:

Theorem 3.3. A set $A$ is $K$-trivial if and only if it is low for $\Omega$ preserving.

We actually want a slight generalization of Stephan and Yu's result.

Lemma 3.4. If $A$ is $K$-trivial and $Y$ is low for $\Omega$, then $Y \equiv_{\mathrm{LR}}(Y \oplus A)$.

Proof. Let $A$ be $K$-trivial and $Y$ be low for $\Omega$. Let $X$ be any $Y$-random. By Theorem 1.3, there is a $Y$-random set $W$ such that both $\Omega$ and $X$ are computable from $W \oplus Y$. There is a nonempty $\Pi_{1}^{0}[Y]$ class containing only members with PA degree relative to $Y$. So by Theorem 1.5, there is a low for $W$ set $S$ with PA degree relative to $Y$. Thus $W$ is $S$-random and $Y \leq_{\mathrm{T}} S$. By Theorem 1.4, both $X$ and $\Omega$ are also $S$-random. Since $S$ is PA and low for $\Omega$, by Theorem 1.2, $S$ computes every $K$-trivial. In particular, $A \leq_{\mathrm{T}} S$. Because $Y \oplus A \leq_{\mathrm{T}} S$ and $X$ is $S$-random, $X$ is $Y \oplus A$-random. But $X$ was any $Y$-random set, so $Y \equiv_{\mathrm{LR}} Y \oplus A$.

The property above is easily seen to imply $K$-triviality, giving us our second characterization of $K$-triviality and answering a question of Merkle (see [11]).

Theorem 3.5. A set $A$ is $K$-trivial if and only if for all $Y$

$$
Y \text { is low for } \Omega \Longrightarrow Y \equiv_{\mathrm{LR}}(Y \oplus A) \text {. }
$$


Proof. One direction is Lemma 3.4. For the other direction, assume that $A$ has the given property. Note $\Omega$ is $\emptyset$-random, so $\emptyset \equiv_{\mathrm{LR}} \emptyset \oplus A \equiv_{\mathrm{LR}} A$. In other words, $A$ is low for randomness, hence $K$-trivial (see Theorem 1.1).

It is natural to ask if low for $X$ preserving is equivalent to $K$-triviality for all random $X$. As we shall see, this is not the case, though it is true for some $X$.

Proposition 3.6. If $\Omega \leq_{\mathrm{T}} X$, then low for $X$ preserving is equivalent to $K$ triviality.

Proof. One direction is given by Proposition 3.2. For the other direction, let $A$ be $K$-trivial and take any $Y$ such that $X$ is $Y$-random. By (the unrelativized form of) Theorem 1.4, $\Omega$ is also $Y$-random. By Lemma 3.4, $Y \equiv_{\mathrm{LR}}(Y \oplus A)$. Therefore, $X$ is $(Y \oplus A)$-random.

For other $X$, low for $X$ preserving is equivalent to being computable.

Proposition 3.7. If $X$ is Schnorr $\left[\emptyset^{\prime}\right]$ random but not 2-random, then only the computable sets are low for $X$ preserving.

Proof. We prove the contrapositive. Assume that $A$ is not computable. If $A$ is not $\Delta_{2}^{0}$, then it is not $K$-trivial, hence by Proposition 3.2, it is not low for $X$ preserving. So assume that $A$ is $\Delta_{2}^{0}$. By Posner-Robinson [12], there is a low set $Y$ such that $Y \oplus A \equiv_{\mathrm{T}} \emptyset^{\prime}$. Because $X$ is Schnorr $\left[\emptyset^{\prime}\right]$, it is random relative to any low set, ${ }^{4}$ so it is $Y$-random. But $X$ is not 2-random, so it is not $(Y \oplus A)$-random. Therefore, $A$ is not low for $X$ preserving.

\section{REFERENCES}

[1] Gregory J. Chaitin. Nonrecursive infinite strings with simple initial segments. IBM Journal of Research and Development, 21:350-359, 1977.

[2] Rod Downey, Denis R. Hirschfeldt, Joseph S. Miller, and André Nies. Relativizing Chaitin's halting probability. J. Math. Log., 5(2):167-192, 2005.

[3] Rodney G. Downey and Denis R. Hirschfeldt. Algorithmic randomness and complexity. Theory and Applications of Computability. Springer, New York, 2010.

[4] Péter Gács. Every sequence is reducible to a random one. Inform. and Control, 70(2-3):186192, 1986.

[5] Denis R. Hirschfeldt, André Nies, and Frank Stephan. Using random sets as oracles. J. Lond. Math. Soc. (2), 75(3):610-622, 2007.

[6] Bjørn Kjos-Hanssen. Low for random reals and positive-measure domination. Proc. Amer. Math. Soc., 135(11):3703-3709, 2007.

[7] Antonín Kučera. Measure, $\Pi_{1}^{0}$-classes and complete extensions of PA. In Recursion theory week (Oberwolfach, 1984), volume 1141 of Lecture Notes in Math., pages 245-259. Springer, Berlin, 1985.

[8] Joseph S. Miller and Liang Yu. On initial segment complexity and degrees of randomness. Trans. Amer. Math. Soc., 360(6):3193-3210, 2008.

[9] André Nies. Lowness properties and randomness. Adv. Math., 197(1):274-305, 2005.

[10] André Nies. Computability and randomness, volume 51 of Oxford Logic Guides. Oxford University Press, Oxford, 2009.

[11] André Nies (editor). Logic Blog 2014. ???

[12] David B. Posner and Robert W. Robinson. Degrees joining to 0' '. J. Symbolic Logic, 46(4):714$722,1981$.

[13] Jan Reimann and Theodore A. Slaman. Measures and their random reals. To appear in the Transactions of the American Mathematical Society.

\footnotetext{
${ }^{4}$ In fact, this property characterizes Schnorr $\left[\emptyset^{\prime}\right]$ randomness: Yu [17] showed that $X$ is Schnorr $\left[\emptyset^{\prime}\right]$ random if and only if $X$ is $Z$-random for every low set $Z$.
} 
[14] Stephen G. Simpson and Frank Stephan. Cone avoidance and randomness preservation. To appear in the Annals of Pure and Applied Logic.

[15] Robert M. Solovay. Draft of paper (or series of papers) on Chaitin's work. Unpublished notes, 215 pages, May 1975.

[16] Michiel van Lambalgen. The axiomatization of randomness. J. Symbolic Logic, 55(3):1143$1167,1990$.

[17] Liang Yu. Characterizing strong randomness via Martin-Löf randomness. Ann. Pure Appl. Logic, 163(3):214-224, 2012.

(Greenberg) School of Mathematics, Statistics and Operations Research, Victoria University of Wellington, Wellington, New Zealand

E-mail address: greenberg@msor.vuw.az.nz

(Miller) Department of Mathematics, University of Wisconsin, Madison, Wi 537061388, USA

E-mail address: jmiller@math.wisc.edu

(Monin) School of Mathematics, Statistics and Operations Research, Victoria UniVersity of Wellington, Wellington, NeW Zealand

E-mail address: benoit.monin@computability.fr

(Turetsky) Kurt Gödel Research Center, University of Vienna, Vienna, Austria

E-mail address: turetsd4@univie.ac.at 